\title{
CERTAIN PROPERTIES OF RATIONAL FUNCTIONS INVOLVING BESSEL FUNCTIONS
}

\author{
R. AGHALARY, A. EBADIAN AND Z. OROUJI
}

\begin{abstract}
Let $g_{v}(z)$ be the classical Bessel function of the first kind of order $v$ and $f$ be an analytic function defined on the unit disc $\Delta$. Suppose the operator $H(f)$ be defined by $H(f)(z)=\frac{z}{\frac{z}{f(z)} * \frac{g g_{v}(z)}{z}}$. In this paper we identify subfamily $M_{n}(\alpha, \beta)$ of univalent functions and obtain conditions on the parameter $v$ such that $f \in M_{n}(\alpha, \beta)$ implies $H(f) \in$ $M_{n}(\alpha, \beta)$.
\end{abstract}

\section{Introduction and preliminaries}

Let $\mathscr{A}_{n}$ be the class of functions $f(z)$ normalized in the form

$$
f(z)=z+\sum_{k=n+1}^{\infty} a_{k} z^{k} \quad(n \in \mathbb{N}=\{1,2, \ldots\})
$$

which are analytic in the open unit disc $\Delta=\{z:|z|<1\}$. As usual, we denote by $S$ the subclass of $\mathscr{A}_{n}$, consisting of functions which are univalent in $\Delta$.

We denote by $M_{n}(\alpha, \beta), \alpha>1, \beta \leq 0$ the subclass of $S$ consisting of functions $f(z) \in S$ which satisfy the inequality:

$$
R e\left(\frac{z f^{\prime}(z)}{f(z)}\right) \leq \beta\left|\frac{z f^{\prime}(z)}{f(z)}-1\right|+\alpha,(z \in \Delta) .
$$

For $n=1, \beta=0$ and $1<\alpha<\frac{4}{3}$, the class $M_{1}(\alpha, 0)$ was investigated earlier by Uralegaddi et al. (cf [14]; see also [1], [9] and [11].). Also the classes $M_{1}(\alpha, \beta)$ and $M_{n}(\alpha, 0)$ were studied by Nishiwaki and Owa [10] and Owa et all [8].

The Bessel function of the first kind of order $v$ is defined by the infinite series

$$
J_{v}(z)=\sum_{n=0}^{\infty} \frac{(-1)^{n}\left(\frac{z}{2}\right)^{2 n+v}}{n ! \Gamma(n+v+1)}
$$

Corresponding author: R. Aghalary. 2010 Mathematics Subject Classification. Primary 30C45; Secondary 30C80.

Key words and phrases. Rational functions, starlike function, Bessel functions, convolution. 
where $\Gamma$ stands for the Euler gamma function, $z \in \mathbb{C}$ and $v \in \mathbb{R}$. Recently, Szász and Kupán [12] investigated the univalence of the normalized Bessel function of the first kind $g_{v}: \Delta \rightarrow \mathbb{C}$, defined by

$$
g_{v}(z)=2^{v} \Gamma(v+1) z^{1-\frac{v}{2}} J_{v}\left(z^{\frac{1}{2}}\right)=z+\sum_{n=1}^{\infty} \frac{(-1)^{n} z^{n+1}}{4^{n} n !(v+1) \cdots(v+n)} .
$$

Baricz [4] and Baricz and Frasin [7] derived several sufficient conditions for integral operators defined by Bessel functions of the first kind to be convex and also proved univalency of integral operators involving Bessel functions. Also we refer the reader for more information about Bessel functions to $[2,3,5,6]$.

In this paper we shall be mainly concerned with functions $f(z)=z+\sum_{k=n+1}^{\infty} a_{k} z^{k}$ of the form

$$
\frac{z}{f(z)}=1-\sum_{k=n}^{\infty} b_{k} z^{k}, \quad z \in \Delta .
$$

It is easy to see that $M_{n}\left(\alpha, \beta_{1}\right) \subset M_{n}\left(\alpha, \beta_{2}\right)$ for $\beta_{1} \leq \beta_{2} \leq 0$ and $M_{n}\left(\alpha_{1}, \beta\right) \subset M_{n}\left(\alpha_{2}, \beta\right)$ for $1 \leq$ $\alpha_{1} \leq \alpha_{2}$.

We obtain necessary and sufficient conditions for functions of the form (1.1) to be in the class $M_{n}(\alpha, \beta)$. Then we find some sufficient conditions for functions of the form $\frac{z^{2}}{g_{v}(z)}$ to be in the class $M_{n}(\alpha, \beta)$. Also we obtain conditions for functions defined by $\frac{z}{\frac{z}{f(z)} * \frac{g_{v}(z)}{z}}$ to be in the class $M_{n}(\alpha, \beta)$.

\section{Main results}

We start with following theorem for which states coefficient conditions for functions in the $M_{n}(\alpha, \beta)$.

Theorem 2.1. Let $\alpha>1, \beta \leq 0$. If a function of the form (1.2), with $b_{n} \geq 0$ belongs to $M_{n}(\alpha, \beta)$, then we have

$$
\sum_{k=n}^{\infty}[k(1-\beta)+(\alpha-1)] b_{k} \leq \alpha-1 .
$$

Proof. Suppose $f \in M_{n}(\alpha, \beta)$. It is easy to check that

$$
z \frac{d}{d z}\left(\frac{z}{f(z)}\right)=\frac{z}{f(z)}-\left(\frac{z}{f(z)}\right)\left(\frac{z f^{\prime}(z)}{f(z)}\right) .
$$

Using the identity (2.2), we have

$$
\operatorname{Re}\left(\frac{z f^{\prime}(z)}{f(z)}\right) \leq \beta\left|\frac{z f^{\prime}(z)}{f(z)}-1\right|+\alpha,
$$

if and only if

$$
\operatorname{Re}\left(1-z \frac{\frac{d}{d z}\left(\frac{z}{f(z)}\right)}{\frac{z}{f(z)}}\right) \leq \beta\left|-z \frac{\frac{d}{d z}\left(\frac{z}{f(z)}\right)}{\frac{z}{f(z)}}\right|+\alpha
$$


The last inequality is equivalent to

$$
1+\operatorname{Re}\left(\frac{\sum_{k=n}^{\infty} k b_{k} z^{k}}{1-\sum_{k=n}^{\infty} b_{k} z^{k}}\right) \leq \beta\left|\frac{\sum_{k=n}^{\infty} k b_{k} z^{k}}{1-\sum_{k=n}^{\infty} b_{k} z^{k}}\right|+\alpha .
$$

If $z \in \Delta$ is real and tends to $1^{-}$through reals, then from (2.3) we obtain

$$
1-\alpha+\left(\frac{\sum_{k=n}^{\infty} k b_{k}}{1-\sum_{k=n}^{\infty} b_{k}}\right) \leq \beta \frac{\sum_{k=n}^{\infty} k b_{k}}{1-\sum_{k=n}^{\infty} b_{k}} .
$$

and the desired assertion follows from the above inequality.

Theorem 2.2. Suppose that $1<\alpha \leq 2$ and $\beta \leq 0$. Let $\frac{z}{f(z)}$ be a nonvanishing analytic function of the form (1.2). Then the following condition is sufficient for $f$ to be in the class $M_{n}(\alpha, \beta)$.

$$
\sum_{k=n}^{\infty}[k(1-\beta)+(\alpha-1)]\left|b_{k}\right| \leq \alpha-1
$$

Proof. As in the proof of Theorem 2.1, we note that the condition

$$
\operatorname{Re}\left(\frac{z f^{\prime}(z)}{f(z)}\right) \leq \beta\left|\frac{z f^{\prime}(z)}{f(z)}-1\right|+\alpha,
$$

is equivalent to

$$
\operatorname{Re}\left(\frac{\sum_{k=n}^{\infty} k b_{k} z^{k}}{1-\sum_{k=n}^{\infty} b_{k} z^{k}}\right) \leq \beta\left|\frac{\sum_{k=n}^{\infty} k b_{k} z^{k}}{1-\sum_{k=n}^{\infty} b_{k} z^{k}}\right|+(\alpha-1)
$$

or

$$
\operatorname{Re}\left(1-\alpha+\omega\left(1-\beta e^{i \theta}\right)\right) \leq 0
$$

where

$$
-\pi \leq \theta<\pi \quad \text { and } \quad \omega=\frac{\sum_{k=n}^{\infty} k b_{k} z^{k}}{1-\sum_{k=n}^{\infty} b_{k} z^{k}} .
$$

Using the fact $R e W \leq 0$ if and only if $|1+W| \leq|1-W|$, it suffices to show that $A \leq B$ where

$$
A=\left|(1+(1-\alpha))\left(1-\sum_{k=n}^{\infty} b_{k} z^{k}\right)+\sum_{k=n}^{\infty} k b_{k} z^{k}-\sum_{k=n}^{\infty} k b_{k} \beta e^{i \theta} z^{k}\right|
$$

and

$$
B=\left|(1-(1-\alpha))\left(1-\sum_{k=n}^{\infty} b_{k} z^{k}\right)-\sum_{k=n}^{\infty} k b_{k} z^{k}+\sum_{k=n}^{\infty} k b_{k} \beta e^{i \theta} z^{k}\right|
$$

From the hypothesis we can write

$$
A-B \leq|2-\alpha|+\sum_{k=n}^{\infty}(k-1-(1-\alpha))\left|b_{k}\right|-\beta \sum_{k=n}^{\infty} k\left|b_{k}\right|
$$




$$
-|\alpha|+\sum_{k=n}^{\infty}(1+k-(1-\alpha))\left|b_{k}\right|-\beta \sum_{k=n}^{\infty} k\left|b_{k}\right| .
$$

For $1<\alpha \leq 2$, we have

$$
A-B \leq \sum_{k=n}^{\infty}(2 k(1-\beta)-2(1-\alpha))\left|b_{k}\right|-2(\alpha-1) .
$$

The last expression is non-positive by (2.4) and so $f$ is in the class $M_{n}(\alpha, \beta)$.

By proceeding the same as proof of Theorem 2.1 we can get the following result in which we omit the details.

Theorem 2.3. Let $f(z)=z+\sum_{n=2}^{\infty} a_{n} z^{n}$ with $a_{n} \geq 0$ belongs to $M_{1}(\alpha, \beta)$, then

$$
\sum_{n=2}^{\infty}[n(1-\beta)+(\beta-\alpha)] a_{n} \leq \alpha-1
$$

Corollary 2.1. Suppose $-1 \leq \beta \leq 0$ and $1<\alpha<\frac{4-\beta}{3}$. If $f(z)=z+\sum_{n=2}^{\infty} a_{n} z^{n}$ with $a_{n} \geq 0$ belongs to $M_{1}(\alpha, \beta)$, then $f(z) \in S^{*}\left(\frac{4-\beta-3 \alpha}{3-\beta-2 \alpha}\right)$.

Proof. By Theorem 2.3 for $f(z)=z+\sum_{n=2}^{\infty} a_{n} z^{n} \in M_{1}(\alpha, \beta)$ with $a_{n} \geq 0$ we have

$$
\sum_{n=2}^{\infty} a_{n}[n(1-\beta)+(\beta-\alpha)] \leq \alpha-1 .
$$

It is well-known if $f(z) \in \mathscr{A}_{1}$ satisfies

$$
\sum_{n=2}^{\infty} \frac{n-\gamma}{1-\gamma}\left|a_{n}\right| \leq 1
$$

for some $\gamma(0 \leq \gamma<1)$, then $f(z) \in s^{*}(\gamma)$ by Silverman [13]. Therefore, we have to find the smallest positive $\gamma$ such that

$$
\sum_{n=2}^{\infty} \frac{n-\gamma}{1-\gamma}\left|a_{n}\right| \leq \sum_{n=2}^{\infty} \frac{[n(1-\beta)+(\beta-\alpha)]}{\alpha-1}\left|a_{n}\right| \leq 1
$$

This gives that

$$
\gamma \leq \frac{n(2-\beta-\alpha)+(\beta-\alpha)}{n(1-\beta)+1+\beta-2 \alpha},
$$

for all $n=2,3,4, \ldots$. Nothing that the right side of the above inequality is increasing for $n$, we conclude that

$$
\gamma \leq \frac{4-\beta-3 \alpha}{3-\beta-2 \alpha}
$$

which proves that $f(z) \in S^{*}\left(\frac{4-\beta-3 \alpha}{3-\beta-2 \alpha}\right)$.

By combining Theorems 2.1 and 2.2 we have 
Corollary 2.2. Let $\frac{z}{f(z)}$ be a nonvanishing analytic function in $\Delta$ of the form $\frac{z}{f(z)}=1-\sum_{k=n}^{\infty} b_{k} z^{k}$ with $1<\alpha \leq 2$ and $b_{k} \geq 0$ for $k \geq n$. Then $f$ is in the class $M_{n}(\alpha, \beta)$ if and only if

$$
\sum_{k=n}^{\infty}[k(1-\beta)+(\alpha-1)] b_{k} \leq(\alpha-1) .
$$

Example 2.1. Let $1<\alpha \leq 2$ and $\beta \leq 0$. Then the function $f(z)$ given by

$$
f(z)=\frac{(m(1-\beta)+(\alpha-1)) z}{m(1-\beta)+(\alpha-1)\left(1-z^{m}\right)}, \quad m \geq n
$$

belongs to the class $M_{n}(\alpha, \beta)$.

Theorem 2.4. Let $1<\alpha \leq 2, \beta \leq 0$ and $g_{v}(z)$ be defined by (1.1). Then the following condition is sufficient for $f(z)=\frac{z^{2}}{g_{v}(z)}$ to be in the class $M_{1}(\alpha, \beta)$

$$
\frac{(1-\beta)}{4(v+1)}(1+M(v+1))+(\alpha-1) M(v) \leq \alpha-1, \quad v>-1
$$

where $M(v)=\sum_{k=1}^{\infty} \frac{1}{4^{k} k !(v+1)_{k}}$.

Proof. Set $\frac{z}{f(z)}=\frac{g_{v}(z)}{z}$. It is well-known that the function $\frac{g_{v}(z)}{z}$ is univalent for $v>-1$ and so is nonvanishing analytic function on $\Delta$. Hence by using Theorem 2.2, it suffices to show that

$$
P:=\sum_{k=1}^{\infty} \frac{k(1-\beta)+(\alpha-1)}{4^{k} k !(v+1) \cdots(v+k)} \leq \alpha-1 .
$$

But by the hypotheses and (2.7) it is easy to see that

$$
\begin{aligned}
P & =\frac{1-\beta}{4(v+1)} \sum_{k=1}^{\infty} \frac{1}{4^{k-1}(k-1) !(v+2)_{k-1}}+(\alpha-1) \sum_{k=1}^{\infty} \frac{1}{4^{k} k !(v+1)_{k}} \\
& =\frac{(1-\beta)}{4(v+1)}(1+M(v+1))+(\alpha-1) M(v) \leq \alpha-1
\end{aligned}
$$

This complete the proof.

Remark 2.1. For $v>-1$, it is easy to check that

$$
(v+1)(v+2) \cdots(v+k) \geq(v+1)^{k} \quad k=1,2,3, \ldots
$$

Therefore

$$
M(v)=\sum_{k=1}^{\infty} \frac{1}{4^{k} k !(v+1)_{k}} \leq \sum_{k=1}^{\infty} \frac{1}{4^{k} k !(v+1)^{k}}=e^{\frac{1}{4(v+1)}}-1,
$$

also

$$
\frac{1}{4(v+1)}(M(v+1)+1)=\sum_{k=1}^{\infty} \frac{1}{4^{k}(k-1) !(v+1)_{k}}
$$




$$
\begin{aligned}
& =\frac{1}{4(v+1)}+\frac{1}{4(v+1)} \sum_{k=1}^{\infty} \frac{1}{4^{k} k !(v+2)_{k}} \\
& \leq \frac{1}{4(v+1)}\left(1+\sum_{k=1}^{\infty} \frac{1}{4^{k} k !(v+2)^{k}}\right) \\
& =\frac{1}{4(v+1)} e^{\frac{1}{4(v+2)}}
\end{aligned}
$$

implies that we can reduce the conditions of Theorem 2.4 as follows:

Corollary 2.3. If $\beta \leq 0,1<\alpha \leq 2$ and $v>-1$ satisfy the condition

$$
\frac{1-\beta}{4(v+1)} e^{\frac{1}{4(v+2)}}+(\alpha-1)\left(e^{\frac{1}{4(v+1)}}-1\right) \leq(\alpha-1),
$$

then $\frac{z^{2}}{g_{v}(z)} \in M_{1}(\alpha, \beta)$.

Theorem 2.5. Let $1<\alpha \leq 2, \beta \leq 0$ and $f, g \in M_{n}(\alpha, \beta)$ be of the form $\frac{z}{f(z)}=1-\sum_{k=n}^{\infty} b_{k} z^{k}$ and $\frac{z}{g(z)}=1-\sum_{k=n}^{\infty} c_{k} z^{k}$ with $b_{k} \geq 0, c_{k} \geq 0$ for all $k \geq n$. Also let $\frac{z}{f(z)} * \frac{z}{g(z)} \neq 0$ on $\Delta$. Then the function $H(z)$ defined by

$$
H(z)=\frac{z}{\frac{z}{f(z)} * \frac{z}{g(z)}}
$$

belongs to $M_{n}(\alpha, \beta)$.

Proof. By Theorem 2.2 it is sufficient to show that

$$
\sum_{k=n}^{\infty}[k(1-\beta)+(\alpha-1)] b_{k} c_{k} \leq \alpha-1 .
$$

Since $f, g \in M_{n}(\alpha, \beta)$, by Theorem 2.1 we have

$$
\sum_{k=n}^{\infty}[k(1-\beta)+(\alpha-1)] b_{k} \leq \alpha-1,
$$

and

$$
\sum_{k=n}^{\infty}[k(1-\beta)+(\alpha-1)] c_{k} \leq \alpha-1 .
$$

Using the Schwarz's inequality, with relations (2.9) and (2.10) we obtain

$$
\sum_{k=n}^{\infty}[k(1-\beta)+(\alpha-1)] \sqrt{b_{k} c_{k}} \leq \alpha-1 .
$$

By the hypotheses and (2.11) it follows that

$$
\begin{aligned}
& \sum_{k=n}^{\infty}[k(1-\beta)+(\alpha-1)] b_{k} c_{k} \\
& \leq \sum_{k=n}^{\infty}[k(1-\beta)+(\alpha-1)] \sqrt{b_{k} c_{k}} \\
& \leq \alpha-1,
\end{aligned}
$$

and the proof is complete. 
Theorem 2.6. Let $\beta \leq 0,1<\alpha \leq 2$ and $f \in M_{n}(\alpha, \beta)$ have the form $\frac{z}{f(z)}=1-\sum_{k=n}^{\infty} b_{k} z^{k}$ with $b_{k} \geq 0$, for all $k \geq n$. Suppose that $g(z)=1+\sum_{k=n}^{\infty} c_{k} z^{k}$ is an analytic function in $\Delta$ with $\left|c_{k}\right| \leq 1$ for all $k \geq n$ and such that $\frac{z}{f(z)} * g(z) \neq 0$ on $\Delta$. Then the function $H(z)$ defined by

$$
H(z)=\frac{z}{\frac{z}{f(z)} * g(z)}
$$

is in the class $M_{n}(\alpha, \beta)$.

Proof. By hypotheses, we have $\frac{z}{H(z)} \neq 0$ for $z \in \Delta$. Since

$$
\sum_{k=n}^{\infty}[k(1-\beta)+(\alpha-1)]\left|b_{k} c_{k}\right| \leq \sum_{k=n}^{\infty}[k(1-\beta)+(\alpha-1)] b_{k} \leq \alpha-1,
$$

by Theorem 2.2 we conclude that $H(z) \in M_{n}(\alpha, \beta)$.

Corollary 2.4. Let $\beta \leq 0,1<\alpha \leq 2$ and $f \in M_{n}(\alpha, \beta)$ have the form $\frac{z}{f(z)}=1-b_{n} z^{n}-b_{n+1} z^{n+1}-$ $\cdots$ and $b_{k} \geq 0$ for all $k \geq n$. Suppose that $v>-1$ and $\frac{z}{f(z)} * \frac{g_{v}(z)}{z} \neq 0$ on $\Delta$. Then the function $H(z)$ defined by

$$
H(z)=\frac{z}{\frac{z}{f(z)} * \frac{g_{v}(z)}{z}}
$$

is in the class $M_{n}(\alpha, \beta)$.

Proof. In view of Theorem 2.6 it is sufficient to show that

$$
4^{k} k !(v+1) \cdots(v+k) \geq 1 \quad(k \geq n),
$$

and this is always true.

\section{References}

[1] R. M. Ali, M. Hussain, V. Ranichandran and K. G. Subramanian, A class of multivalent functions with positive coefficients defined by convolution, J. Ineq. Pure. Appl. Math., 6(1)(2005), 1-9.

[2] Á. Baricz, Geometric properties of generalized Bessel functions of complex order, Mathematica, 4871(2006), $13-18$.

[3] Á. Baricz, Bessel transforms and Hardy space of generalized Bessel functions, Mathematica, 4871(2006), 127136.

[4] Á. Baricz, Geometric properties of generalized Bessel functions, Publ. Math. Debrecen., 73(2008), 155-178.

[5] Á. Baricz, Generalized Bessel functions of the first kind, Lecture Notes in Mathematics, Vol. 1994, SpringerVerlag, Berlin, 2010.

[6] Á. Baricz and S. Ponnusamy, Starlikeness and convexity of generalized Bessel functions, Integral Transforms Spec. Funct., 21(2010), 641-653.

[7] Á. Baricz and A. Frasin, Univalence of integral operators involving Bessel functions, Appl. Math. Lett., 23(2010), 371-376. 
[8] S. Owa , M. Nunokawa and H. M. Srivastava, A certain class of multivalent functions, Appl. Math. Lett., 10(2) (1997), 7-10.

[9] J. Nishiwaki and S. Owa, Coefficient inequalities for analytic functtions, Int. J. Math. Math. Sci., 29(2002), 285-290.

[10] J. Nishiwaki and S. Owa, Certain classes of analytic functions concerned with uniformly starlike and convex functions, Applied. Math. and Computation, 187(2007), 350-357.

[11] S. Owa and J. Nishiwaki, Coefficient estimates for certain classes of analytic functions, J. Ineq. Pure. Appl. Math., 3(5) (2002), 1-5.

[12] R. Szász, P. Kupán, About the univalence of the Bessel functions, Stud. Univ. Babes-Bolyai Math., 54(1)(2009), 127-132.

[13] H. Silverman, Univalent functions with negative coefficients, Proc. Amer. Math. Soc., 51(1975), $109-116$.

[14] B. A. Uralegaddi, M. D. Ganigi and S. A. Sarangi, Univalent functions with positive coefficients, Tamkang J. Math., 25(1994), 225-290.

Department of Mathematics, Faculty of Science, Urmia University, Urmia, Iran.

E-mail: raghalary@yahoo.com

Department of Mathematics, Faculty of Science, Urmia University, Urmia, Iran.

E-mail: a.ebadian@urmia.ac.ir

Department of Mathematics, Faculty of Science, Urmia University, Urmia, Iran.

E-mail: z.oroujy@yahoo.com 